\title{
Influence of pulling handles and device stiffness in single-molecule force spectroscopy
}

\author{
Arijit Maitra and Gaurav Arya* \\ Received 18th August 2010, Accepted 15th September 2010 \\ DOI: $10.1039 / \mathrm{c0cp01528h}$
}

In single-molecule force spectroscopy, individual molecules and complexes are often stretched by pulling devices via intervening molecular handles. Accurate interpretation of measurements from such experiments in terms of the underlying energy landscape, defined by activation barriers and intrinsic rates of transition, relies on our understanding, and proper theoretical treatment, of the effects of the pulling device and handle. Here, we present a framework based on Kramers' theory that elucidates the dependence of measured rupture forces and rates on the pulling device stiffness and attributes of the handle, contour length and persistence length. We also introduce a simple analytic model that improves prediction of activation barriers and intrinsic rates for all device stiffnesses and handle properties, thus allowing for a more reliable interpretation of experiments. Our analyses also suggests intuitive ways of displaying the measured force spectra for proper prognosis of device and handle effects and provides the range of device and handle attributes over which these effects can be neglected.

\section{Introduction}

Single-molecule force spectroscopy ${ }^{1-3}$ (SMFS) has attracted much attention in recent times for its ability to unravel complex biomolecular interactions and reaction-pathways. ${ }^{4,5}$ In these techniques, tiny forces ranging from pico- to nanonewtons are applied to molecules of interest using sophisticated devices like optical traps and AFM cantilevers to record their response to stretching. Molecular response is generally characterized in two ways - distribution of lifetime $p(\tau)$ of bound molecular conformation at different fixed force levels $F$ ("constant-force" experiments) and distribution of forces $p\left(F_{R}\right)$ at which molecules rupture under the action of linearly or quasi-linearly increasing force levels ("constant-speed" experiments).

The underlying microscopic interactions governing molecular response is best understood in terms of the free energy landscape, which provides information about the stabilities of molecular conformations, barriers to activation, range of interactions, and kinetics of transition between states. Understanding this link between molecular response and the energy landscape has been greatly facilitated by theoretical efforts. ${ }^{6-11}$ Improved statistical interpretation of the measurements have allowed extraction of the energy landscape parameters - the spontaneous rate of rupture $k_{0}$, the activation energy barrier $U^{*}$, and distance of the barrier from the native state $x^{*}$. The earliest work provided an asymptotic relationship between rupture rate $k(F)$ and force $F$ for small applied forces. ${ }^{6}$ Subsequently, the kinetics of force-induced bond rupture was explained in terms of the diffusion of a Brownian particle ${ }^{9}$ over a continually decreasing barrier by invoking the Kramers' theory. ${ }^{12,13}$ Recently, theoretical models using predefined, one-dimensional

Department of NanoEngineering, University of California, San Diego, 9500 Gilman Dr., La Jolla, CA 92093-0448, USA.

E-mail: garya@ucsd.edu; Fax: 858-534-9553; Tel: 858-822-5542 and single-barrier energy profiles have provided specific yet more realistic descriptions of $k(F), p\left(F_{R}\right)$, and the force spectra $\overline{F_{R}}(\dot{F})\left(\overline{F_{R}}\right.$ : mean rupture force, $\dot{F}$ : loading rate) applicable over a broad range of forces and loading rates, respectively. ${ }^{14-17}$

The above models have been derived for the simplified case of a molecule being pulled directly by a soft device. ${ }^{18}$ However, in reality, particularly to disrupt strongly bound or folded molecules one is required to employ a stiff device (large spring constant). Further, a polymeric handle (also known as a tether, anchor, spacer, or linker) connecting the molecule to the device is used for improving the resolution of rupture events and suppressing unwanted, non-specific interactions between the molecules and the device. ${ }^{19}$ Although, the device ${ }^{20-22}$ and handle ${ }^{23-28}$ have been shown to modulate kinetics and binding strength of molecules and complexes, capturing such effects succinctly within an analytical framework for accurate prediction of the key energy-landscape parameters has remained elusive.

Recently, we have explained the effects of device stiffness in the absence of handles within an analytical model ${ }^{18}$ (also see ref. 22) over a broad range of pulling speeds $V$. Incorporating the effect of a handle is significantly more challenging, ${ }^{27-29}$ particularly, due to its non-linear force-extension relationship beyond the small force regime that makes its rate of loading $\dot{F}(F)$ to depend strongly on the applied force. Compare this to the pulling device, which exhibits simple Hookean behavior where $\dot{F}$ remains constant over a range of forces. Thus, reliable interpretation of SMFS measurements is faced with two outstanding issues: How does the pulling device and handle, concurrently, affect force measurements over a broad range of control parameters? How can these effects be modeled analytically?

In this article, we present an approach based on Kramers' theory that permits us to elucidate the influence of the pulling device and handle over a wide range of handle lengths, applied 
forces, and pulling speeds on $k(F), p\left(F_{R}\right), \overline{F_{R}}(\dot{F})$. We also introduce a simple analytical procedure to model $k(F)$ from arbitrary handle attributes and device stiffnesses, which can be utilized to improve the accuracy of the extracted energy landscape parameters. Additionally, our analyses uncover discernable signatures of force-induced rupture rates and force spectra that could be used as indicators of handle and device effects and allows us to formulate conditions where these effects become important.

\section{Model development}

\section{Modeling bond rupture with pulling device and handles}

We treat the rupture of a molecule or bond at equilibrium $(F=0)$ as the thermally-driven escape of a Brownian particle with diffusivity $D$ from a potential energy well, representing its native bound state. The free energy of the bond along the reaction coordinate $x$ is described by a linear-cubic function $^{8,15}$ $U_{0}(x)=F_{c} x-F_{d} x^{3}\left(F_{c}=1.5 U^{*} / x^{*}, F_{d}=2 U^{*} / x^{* 3}\right)$ with an energy barrier of height $\left.\Delta U\right|_{F=0}=U^{*}$ located a distance $\left.\Delta x\right|_{F=0}=x^{*}$ from the native state. Here, the particle position $x$ represents an order parameter defining the state of the bond and can be used for keeping track of the progress of bond rupture. We next consider facilitated rupture of the bond through constant-speed pulling such that quasistatic condition is maintained throughout the process. This condition is realized when the timescale of pulling is much larger than the timescales of relaxation of the molecule, handle and the device. Three different pulling scenarios (Fig. 1) are examined.

Case I (Fig. 1a top) represents the limiting case where the pulling device of stiffness $K$ is connected directly to the bond and no handle is used, i.e., the handle stiffness $K_{h} \rightarrow \infty$. The total free energy of the composite system is therefore given by

$$
U(x ; t)=U_{0}(x)+U_{\mathrm{dev}}(V t-x ; t),
$$

where $U_{\text {dev }}(\lambda ; t)=K \lambda^{2} / 2$ represents the energy contribution of the device when treated as a Hookean spring ${ }^{1}$ of length $\lambda \equiv \lambda(t)$ at time $t$. The speed of pulling is denoted by $V$.

Case II (Fig. 1a middle) represents another limiting condition where the bond is now connected to a handle and pulled
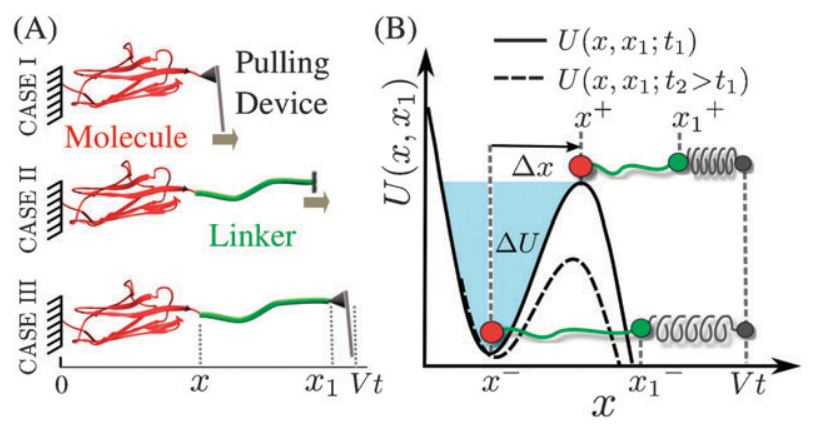

Fig. 1 (a) Cartoon of single-molecule force spectroscopy experiment and the different cases investigated (see text for details). (b) Total free energy profile (solid curve) at time $t=t_{1}\left(F=F_{1}\right)$ is distorted with time $\left(t=t_{2}>t_{1} ; F=F_{2}>F_{1}\right)$ as pulling progresses. Also shown at $t=t_{1}$ are the maximum and minimum energy configurations of the particle, handle and device. without any pulling device, i.e., $K \rightarrow \infty$, the system free energy is then given by

$$
U(x ; t)=U_{0}(x)+U_{\text {han }}(V t-x ; t) .
$$

Here, the handle is treated as a worm-like chain (WLC), ${ }^{30,31}$ which describes the force-extension behavior of doublestranded DNA and unfolded proteins. The WLC energy for a handle end-to-end distance $\lambda \equiv \lambda(t)$ so that $\lambda \geq 0$ at time $t$ is given by

$$
U_{\text {han }}(\lambda ; t)=\frac{1}{2 P \beta}\left[\frac{L}{2\left(1-\frac{\lambda}{L}\right)}+\frac{\lambda^{2}}{L}-\frac{\lambda}{2}\right],
$$

where $P$ and $L$ are the persistence length and contour length, respectively, and $\beta \equiv 1 / k_{\mathrm{B}} T\left(k_{\mathrm{B}}\right.$ : Boltzmann constant and $T$ : temperature).

Case III (Fig. 1a bottom) is the most general case where the bond is connected to the pulling device via an intervening WLC handle with a total free energy given by

$U\left(x, x_{1} ; t\right)=U_{0}(x)+U_{\text {han }}\left(x_{1}-x ; t\right)+U_{\mathrm{dev}}\left(V t-x_{1} ; t\right)$,

where $x$ is the position of the bond connected to one end of the handle and $x_{1}$ is the position of the other end of the handle.

\section{Rupture rate-force relationship}

To compute the instantaneous rate of escape of the Brownian particle from the well for all three cases we use Kramers' theory under the assumption of high barrier $\beta \Delta U \gg 1$ and large particle friction. For case I, we use the exact expression for $k(F):^{18}$

$$
k(F ; \chi)=k_{0}\left(\chi^{2}-\frac{F \chi}{F_{c}}\right)^{\frac{1}{2}} e^{\beta U^{*}\left\{1-\left(\chi^{2}-F \chi / F_{c}\right)^{3 / 2}\right\}},
$$

where $\chi=1+K / K_{m}$ and $K_{m}=\partial^{2} U_{0}(x) /\left.\partial x^{2}\right|_{x}=x^{-}$at the point of energy minimum, $x^{-}$. In the limit of soft pulling device, $\chi=1$, one recovers the relationship given in ref. 15 . We refer to this limit as the soft-spring theory and denote $k^{\text {th }}(F) \equiv k(F ; \chi=1)$. This limit will serve as a baseline for all subsequent comparisons.

Since cases II and III are not analytically tractable, we use a numerical approach to obtain $k(F)$. For case II, we numerically evaluate the time $(t)$ dependent locations of the minimum $x=x^{-}$(the instantaneous state of the bond) and maximum $x=x^{+}$(the instantaneous location of the energy barrier) of the continuously tilting composite energy profile in eqn (2) to yield the energies $U\left(x^{-} ; t\right)$ and $U\left(x^{+} ; t\right)$ and the curvatures $U^{\prime \prime}\left(x^{-} ; t\right)$ and $U^{\prime \prime}\left(x^{+} ; t\right)$. The instantaneous Kramers' escape rate is then computed from

$$
k(t)=\tau(t)^{-1} \exp [-\beta \Delta U(t)]
$$

where $\tau(t)^{-1}=(\beta D / 2 \pi) \sqrt{U^{\prime \prime}\left(x^{-} ; t\right)\left|U^{\prime \prime}\left(x^{+} ; t\right)\right|}$ and $\Delta U(t)=$ $U\left(x^{+} ; t\right)-U\left(x^{-} ; t\right)$. As time is parametrically related to force via $F(t)=K\left(V t-x^{-}\right)$, the time-dependent rupture rates can be easily transformed to force-dependent rupture rates, $k(F)$.

For case III, the composite energy profile is a function of both the particle $x$ and handle position $x_{1}$ (eqn (4)) (see Fig. 1b). However, $x_{1}$ and $x$ are constrained through a quasistatic pulling condition $\partial U\left(x, x_{1} ; t\right) / \partial x_{1}=0$, which stipulates zero net force on the handle at all times. Consequently, 
we can obtain for all $t$ the locations of the minimum $x^{-}(t)$ and maximum $x^{+}(t)$ of $U\left(x, x_{1} ; t\right)$ subject to the constraint $\partial U\left(x, x_{1} ; t\right) / \partial x_{1}=0$ to yield energies $U\left(x^{-}, x_{1}{ }^{-} ; t\right)$ and $U\left(x^{+}, x_{1}{ }^{+} ; t\right)$ and curvatures $U^{\prime \prime}\left(x^{-}, x_{1}{ }^{-} ; t\right)$ and $U^{\prime \prime}\left(x^{+}, x_{1}{ }^{+} ; t\right)$, where $x_{1}{ }^{-}$and $x_{1}{ }^{+}$correspond to values of $x_{1}$ that satisfy the quasistatic pulling constraint for $x=x^{-}$and $x=x^{+}$, respectively. Kramers' theory then provides the rate $k(t)$ (eqn (6)) with $\tau(t)^{-1}=(\beta D / 2 \pi) \sqrt{U^{\prime \prime}\left(x^{-}, x_{1}^{-} ; t\right)\left|U^{\prime \prime}\left(x^{+}, x_{1}^{+} ; t\right)\right|}$ and $\Delta U(t)=U\left(x^{+}, x_{1}{ }^{+} ; t\right)-U\left(x^{-}, x_{1}{ }^{-} ; t\right)$. As in Case II, $k(t)$ can be recast to $k(F)$ via $F(t)=K\left(V t-x_{1}{ }^{-}\right)$.

\section{Distribution of rupture forces}

The distribution of bond rupture times, $p(t)$, and the survival probability $s(t)$ can be computed by substituting $k(t)$ in $p(t)=-\frac{\mathrm{d} s(t)}{\mathrm{d} t}=k(t) s(t) .{ }^{9,32}$ Irrespective of how the applied force scales with time, one can rewrite this as $p(F)=$ $-\mathrm{d} s(F) / \mathrm{d} F=k(F) s(F) / \dot{F}(F)$. Eliminating $s(F)$ from the above equalities, one gets ${ }^{29}$

$$
p\left(F_{R}\right)=\frac{k\left(F_{R}\right)}{\dot{F}} \exp \left\{-\int_{0}^{F_{R}} k(F) / \dot{F}(F) \mathrm{d} F\right\},
$$

where the actual loading rate is denoted by $\dot{F}(F)$ (see eqn (12a)). For case I, the loading rate is constant, $\dot{F}=K V / \chi$, and an exact expression of $p\left(F_{R}\right)$ can be formulated ${ }^{18}$ using eqn (5):

$$
p\left(F_{R}\right)=\frac{k\left(F_{R}\right) e^{q X}}{\dot{F}} \exp \left[-\frac{k\left(F_{R}\right)}{\beta \dot{F} \chi x^{*}}\left(\chi^{2}-\frac{\chi F_{R}}{F_{c}}\right)^{-\frac{1}{2}}\right]
$$

where $q \equiv \exp \left[\beta U^{*}\left\{1-\chi^{3}\right\}\right]$ and $X \equiv k_{0} / \beta \chi \dot{F} x^{*}$. For cases II and III, $p\left(F_{R}\right)$ can only be obtained numerically via eqn (7) by using the numerical force-dependent loading rate $\dot{F} \equiv \mathrm{d} F(t) / \mathrm{d} t$ that includes the effect from WLC.

\section{Mean rupture force versus loading rate}

SMFS data is often displayed by plotting mean rupture forces, $\overline{F_{R}}$, as a function of loading rates, $\dot{F} . \overline{F_{R}}$ is defined as

$$
\overline{F_{R}}=\int_{0}^{\infty} F_{R} p\left(F_{R}\right) \mathrm{d} F_{R} .
$$

For case I, $\dot{F}$ is the force independent loading rate $K V / \chi$ and we use the expression of $\overline{F_{R}}(\dot{F}):^{18}$

$$
\overline{F_{R}} \cong F_{c} \chi\left[1-\left\{1-\frac{e^{q X} E_{1}(q X)}{\beta U^{*} \chi^{3}}\right\}^{2 / 3}\right]
$$

Here $E_{1}(u)=\int_{u}^{\infty} \frac{e^{-z}}{z} \mathrm{~d} z$ is the exponential integral ${ }^{33}$ which can be approximated as $e^{u} E_{1}(u) \approx \ln \left(1+e^{-\gamma} / u\right)$, where $\gamma=0.577 \ldots$ is the Euler-Mascheroni constant. In the limit of soft springs $\chi=1(q=1)$, eqn (10) is identical to the prediction for soft devices. ${ }^{16}$

For cases II and III we find that the definition $\dot{F}=K V$ is permissible only in the limit of soft pulling device. Outside this limit, and in general, one must use the actual loading rate inclusive of the compliance of all the elements, namely, the pulling device, the handle (if present), and the molecule. As the loading rate is dependent on force due to the linker elasticity, we find it intuitive to compute the actual rate of loading as a mean value from

$$
\overline{\dot{F}}=\int_{0}^{\infty} \dot{F}\left(F_{R}\right) p\left(F_{R}\right) \mathrm{d} F_{R}
$$

In this way we can create a map of $\overline{F_{R}}$ and $\overline{\dot{F}}$ for a set of $\{K, L, V\}$ (using eqn (9) and (11)).

Since experimentally it is not always possible to extract the loading rate at the point of rupture one can combine $p\left(F_{R}\right)$ with the theoretical loading rate ${ }^{29}$

$$
\begin{gathered}
\dot{F}(F)=K_{e} V ; K_{e}(F)^{-1}=K_{m}^{-1}+K_{\mathrm{wc}}(F)^{-1}+K^{-1} \\
K_{m}=\frac{6 U^{*}}{x^{* 2}} ; K_{\mathrm{wlc}}(F)=\frac{3+5 \beta F P+8(\beta F P)^{2.5}}{2 \beta L P(1+\beta F P)},
\end{gathered}
$$

with good accuracy, where $K_{e}^{-1}$ is the effective compliance. Note that we have included the intrinsic compliance $1 / K_{m}$ of the molecule that has been mostly omitted in earlier studies owing to the assumptions $K_{m} \gg K$ and $K_{m} \gg K_{h}$.

\section{Implementation details}

The above analyses of $k(F), p\left(F_{R}\right)$, and $\overline{F_{R}}(\overline{\dot{F}})$ for the three pulling scenarios is carried out at temperature $T=300 \mathrm{~K}$ possessing an intrinsic diffusivity of $D=4.2 \times 10^{-9} \mathrm{~nm}^{2} / \mathrm{ps}$. The bond is described by a linear-cubic energy function with parameters $U^{*}=50 \mathrm{~kJ} \mathrm{~mol}^{-1}$ and $x^{*}=0.25 \mathrm{~nm}$, representative of typical non-covalent interactions stabilizing ligand-receptor complexes. The main conclusions of this study are based on these parameters that typify relatively stiff bonds. Our inferences remain unchanged when we chose parameters that exemplify, for instance, bonds with shallow energy well, as long as the device stiffness is maintained smaller than the bond stiffness. We consider the handle to be made of dsDNA with persistence length $P=50 \mathrm{~nm}$. The control parameters are varied within an experimentally accessible range: $V=10^{-10}$, $5 \times 10^{-10}, 10^{-9}, 5 \times 10^{-9}, 10^{-8} \mathrm{~nm} / \mathrm{ps}, K=1,10,20, \ldots, 100$, $200 \mathrm{~kJ} \mathrm{~mol}^{-1} \mathrm{~nm}^{-2}$ (which represent small to medium level stiff AFM tips), and $L=100,200, \ldots, 1000 \mathrm{~nm}$. All numerical codes are written and executed in MATLAB R2010a.

It is instructive to examine if the imposed pulling speeds satisfy the quasistatic pulling assumption invoked earlier. In other words, is the system being pulled at a rate much slower than the slowest relaxation timescale of the system, which in our system corresponds to that of the WLC handle? Evans and Ritchie $^{27}$ suggested that the timescale of the slowest bending mode of a WLC is given by $t_{\mathrm{wlc}}<\eta P^{2} L / k_{\mathrm{B}} T$, where $\eta$ is the solvent viscosity, while the pulling timescale is given by $t_{p}=x_{e} / V$, where $x_{e} \approx(P L)^{1 / 2}$ is the end-to-end distance of the WLC handle. Therefore, the quasistatic pulling condition $t_{p} \gg t_{\text {wlc }}$ yields $\eta P^{3 / 2} L^{1 / 2} V / k_{\mathrm{B}} T \ll 1$. Using $\eta / k_{\mathrm{B}} T \approx 2.45 \times$ $10^{-10} \mathrm{~s} \mathrm{~nm}^{-3}$ for water, it can be shown that this condition is satisfied for all pulling rates used in our study. Since, these pulling rates are representative of experimental pulling rates, we expect that most typical SMFS experiments satisfy the quasistatic pulling condition. However, for much higher pulling speeds, such as those employed in steered molecular dynamics simulations, the quasistatic assumption may no more be valid. In such cases, the interpretation of data 
becomes difficult due to non-equilibrium effects ${ }^{34}$ and one has to resort to other methods of energy landscape reconstruction, such as the Jarzynski approach. ${ }^{35}$

\section{Results and discussion}

\section{Pulling device and handles exhibit distinct signatures in rupture} rate and force plots

Fig. 2a shows the theoretical $k(F)$ for the limiting case I when no handle is present and only the stiffness of the pulling device modulates rupture process; a distinct convergence of $k(F)$ curves for different $K$ values is noted with increasing force. As we have shown previously, ${ }^{18}$ changing the device stiffness from soft to stiff ( $K=1$ to $200 \mathrm{~kJ} \mathrm{~mol}^{-1} \mathrm{~nm}^{-2}$ ) leads to a suppression of rupture rates at the same force level; this "device effect" is especially strong at small forces. The reason is attributed to the heightening of the energy barrier with the attachment of the device, and specifically for $F \rightarrow 0, \Delta U \approx$ $U^{*}+K x^{* 2} / 2$. Since $k(F) \propto \exp (-\beta \Delta U)$, the kinetic rate is suppressed by the factor $q=\exp \left(-\beta K x^{* 2} / 2\right)$. Hence, the device can be ignored only if $\beta K x^{* 2} / 2 \ll 1$, i.e., the limit of soft devices would make $q \rightarrow 1$. Note that as $x^{*}$ continues to decrease with $F$, so does $q$, thereby causing $k(F)$ from distinct $K$ values to converge with increasing $F$.

A similar compliance based reasoning can explain the diverging appearance of $k(F)$ curves for case II (Fig. 2b). For small forces $F<1 / \beta P$ the WLC handle behaves as a random coil polymer with its stiffness satisfying $K_{\mathrm{wlc}} \approx 1 / \beta L P \ll$ $2 / \beta x^{* 2}$. Hence, $L$ dependence of rupture rates is not observed in this regime. However, handle stiffness in the large force

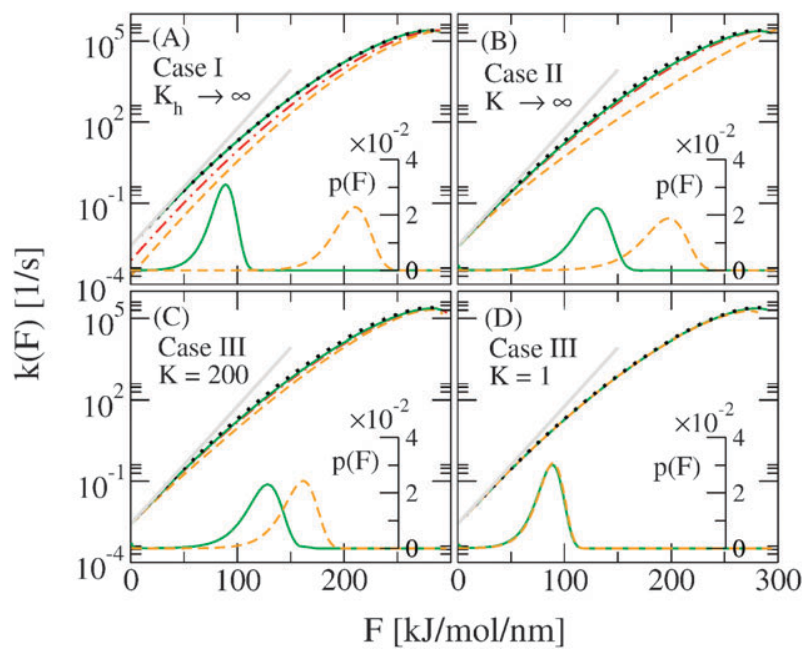

Fig. 2 Rupture rates $k(F)$ versus applied force $F$ at pulling speed $V=10^{-10} \mathrm{~nm} / \mathrm{ps}$ for different conditions: (a) Case I without handle $\left(K_{h} \rightarrow \infty\right)$, (b) Case II with handle but without pulling device $(K \rightarrow \infty)$, and (c,d) Case III with handle and pulling device with $K\left[\mathrm{~kJ} \mathrm{~mol}^{-1} \mathrm{~nm}^{-2}\right]=200$ (c) and $=1$ (d), respectively. In (a) the stiffness are $K\left[\mathrm{~kJ} \mathrm{~mol}^{-1} \mathrm{~nm}^{-2}\right]=1$ (green, solid line), 100 (red, dotdashed line), 200 (orange, dashed line) and in (b-d), the handle lengths are $L[\mathrm{~nm}]=100$ (orange, dashed lines), 500 (red, dot-dashed lines), 1000 (green, solid lines). Bell's relation (gray, solid line) and softspring prediction (black dots) are overlayed. Insets in (a-d) represent the corresponding $p\left(F_{R}\right)$ for each condition.

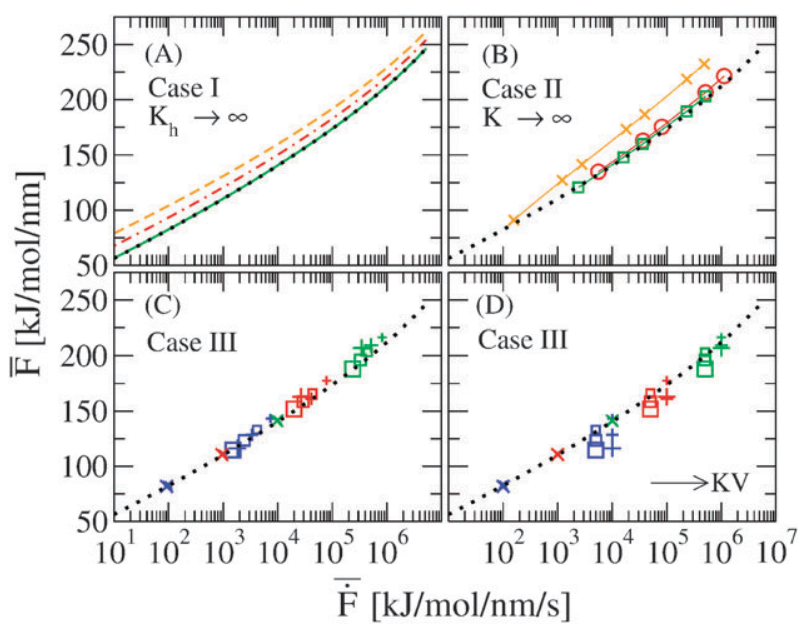

Fig. 3 Mean rupture force versus loading rate for: (a) Case I without handle but pulling device, (b) Case II with handle without pulling device, and (c,d) Case III with handle and pulling device. In $(\mathrm{a}-\mathrm{c})$, the actual loading rate is used while in (d), the apparent loading rate $K V$ is used. For all cases, the soft-spring prediction overlaid as black dots. Symbols: (a) $K\left[\mathrm{~kJ} \mathrm{~mol}^{-1} \mathrm{~nm}^{-1}\right]=1$ (green, solid), 100 (red, dot-dashed), 200 (orange, dashed). (b) Linker length $L=100$ (orange, $\triangle$ ), 500 (red, $\bigcirc$ ), 1000 (green, $\square$ ). (c,d): $K=1(\times), 50(\square), 100(+$ ); symbol size denotes $L[\mathrm{~nm}]=100$ (small), 500 (medium), 1000 (large); and $V[\mathrm{~nm} / \mathrm{ps}]=10^{-10}$ (blue), $10^{-9}$ (red), $10^{-8}$ (green).

limit $F>1 / \beta P$ follows $K_{\text {wlc }} \propto 1 / L$ (from eqn (12)) so that short handles (e.g., $L=100 \mathrm{~nm}$ ) will be less compliant and violate the condition $K_{\mathrm{wlc}} \ll 2 / \beta x^{* 2}$. Hence, at the same high force level, shorter handles will produce greater reduction in the rupture rate than longer handles for constant $P$. Thus, the diverging appearance of the rupture rates versus force is a signature of "handle effects".

Such distinct patterns also emerge in the plots of mean rupture force $\overline{F_{R}}$ versus mean loading rate $\overline{\dot{F}}$ in Fig. $3 \mathrm{a}$ and b. In the former, a slight convergence in $\overline{F_{R}}$ curves from low to high loading rates between the different device stiffnesses is observed while in the latter, a divergence in $\overline{F_{R}}$ curves between the different handle lengths is observed. We propose that such trends, likely also observed in SMFS experiments, could be used to assess whether the device or handle effects need to be ignored or taken into account.

\section{Applicability regime of the soft-spring theory}

The soft-spring theory prediction (eqn (5) with $\chi=1$ ) is overlayed as dotted line in Fig. 2a-d. Clearly, only for sufficiently small $K\left(=1 \mathrm{~kJ} \mathrm{~mol}^{-1} \mathrm{~nm}^{-2}\right.$ ) values (Fig. 2d) do the computed $k(F)$ match those predicted by the soft-spring theory for all handle lengths and a wide range of forces. This is further supported by the overlapping $p\left(F_{R}\right)$ in Fig. 2d-inset for $K=1 \mathrm{~kJ} \mathrm{~mol}^{-1} \mathrm{~nm}^{-2}$ and different $L$. Also, the soft-spring approximation for $\overline{F_{R}}$ versus loading rates (eqn (10) with $\chi=1$ and $q=1$ ) (dotted lines in Fig. $3 \mathrm{c}$ and d) show good fits to the numerical $\overline{F_{R}}$ from $K=1 \mathrm{~kJ} \mathrm{~mol}^{-1} \mathrm{~nm}^{-2}(\times)$ and are independent of $L$ and $V$.

Previously, we have shown that a "soft" device is one that satisfies $K \ll 2 / \beta x^{* 2}$ (assuming that $K \ll K_{m}$, where $K_{m}$ is the native bond stiffness). When both handle and device are 
present, one expects $K_{e} \ll 2 / \beta x^{* 2}$ for the soft-spring theory to be valid. Also, the effective compliance (eqn (12a)) is dominated by the component (molecule, device, or handle) which has the largest compliance. For small forces $F<1 / \beta P$, $K_{\mathrm{wlc}} \approx 1 / \beta L P$ is almost always smaller than $2 / \beta x^{* 2}$. Hence, the soft-spring criteria $K_{e} \ll 2 / \beta x^{* 2}$ is always satisfied. This can be seen in Fig. $2 \mathrm{~b}-\mathrm{d}$ where the computed $k(F)$-curves for all $L$ converge to the soft-spring limit at small forces, irrespective of device stiffness. Interestingly, the asymptotic Bell's model (grey lines, Fig. 2b-d) does an excellent job in this limit where both the handle and device stiffness effects are negligible. On the other hand, for sufficiently large forces $F \gtrsim 1 / \beta P$, and sufficiently small $L$ (see eqn (12b)), one has $K_{\text {wlc }} \gg K$. In this case, the effective compliance is determined solely by $K$. Thus, we conclude that for the entire range of forces, $K \ll 2 / \beta x^{* 2}$ guarantees a unique master curve $k(F)$ for any handle length $L$ and quasi-static pulling speed $V$.

Alternatively, one can formulate a condition for $L$ that guarantees applicability of the soft-spring theory irrespective of $K$, i.e., by choosing a sufficiently long $L$ such that $K_{\mathrm{wlc}}(F) \ll 2 / \beta x^{* 2}$. For large applied forces, this condition becomes $L \gg 2 x^{* 2}(\beta P F)^{1.5} / P$ (from eqn $(12 \mathrm{~b})$ ). Using the maximum possible force in the system $F_{c}=1.5 U^{*} / x^{*}$, the soft-spring theory is then valid for the entire range of forces provided $L \gtrsim 4\left(P x^{*}\right)^{0.5}\left(\beta U^{*}\right)^{1.5}$. In our numerical analysis $L \gtrsim 1300 \mathrm{~nm}$ should lead to a good match between the exact results and the soft-spring theory over a wide range of force irrespective of $K$. Indeed, for $L=1000 \mathrm{~nm}$ in Fig. 2c and 3c, the numerical rupture rates (green, solid line) and rupture forces (large symbols $\times, \square,+$ ) appear close to soft-spring prediction (dotted lines) irrespective of $(K, F)$ and $(K, V)$, respectively. Coincidently, SMFS experiments with optical traps $\left(K \approx 10^{-3}-1 \mathrm{~kJ} \mathrm{~mol}^{-1} \mathrm{~nm}^{-2}\right.$ ) tend to utilize $\mu \mathrm{m}$-long dsDNA handles, ${ }^{4}$ suggesting that the soft-spring theory may be adequate to analyze force measurements from such studies.

\section{Unique master curves and smooth force spectra are not guaranteed for all device stiffnesses and handle lengths}

Even though $p\left(F_{R}\right)$ from constant speed experiments when transformed to $k(F)$ in the limit of soft pulling device or long handles generate a single master curve, this is generally not true for any $K$ and $L$. For instance, in Fig. 2c for $K=200 \mathrm{~kJ} \mathrm{~mol}^{-1} \mathrm{~nm}^{-2}$, different $L$ show incongruence in the intermediate force regime. This incongruence is corroborated by the non-overlapping rupture force distributions for the same $K$ (Fig. 2c-inset) at $L=100 \mathrm{~nm}$ (orange, dashed line) and $1000 \mathrm{~nm}$ (green, solid line) and further accentuated for $K \rightarrow \infty$ results (Fig. 2b-inset).

Since the characteristics of $p\left(F_{R}\right)$ and $k(F)$ are passed onto the force spectra, an increase in $K$ also leads to a scatter in the mean rupture forces $\overline{F_{R}}$ for a given $V$ and different $L$ such that small $L$ values correlate with larger rupture forces and vice versa (Fig. 3d). A similar scatter in $\overline{F_{R}}$ has also been observed experimentally in the rupture of biotin-streptavidin complexes, ${ }^{20}$ also likely originating from variations in the employed $K$ and $L$. As expected, no scatter in $\overline{F_{R}}$ is observed for small $K\left(=\mathrm{kJ} \mathrm{mol}^{-1} \mathrm{~nm}^{-2}\right)$ for all $L$ and $V$, as shown by symbol $\times$ in Fig. $3 d$. These results suggest that only when the device stiffness satisfies $K \ll 2 / \beta x^{* 2}$, the nominal loading rate
$\dot{F}=K V$ acts as a reliable control parameter. This explains why many studies performed with biomembrane force probes and optical tweezers of very small stiffness could successfully use the nominal loading rate to produce unique curves. However, for arbitrary $K$ and $L$, the nominal loading rate is not a good control parameter, as it is not known a priori if the soft-spring limit is satisfied. Thus, the actual loading rate must be used for accurate data analyses. Indeed, when the above spectra is re-plotted with the actual loading rates from eqn (11) (Fig. 3c), the aforementioned scatter in data vanishes.

It is to be noted that a numerical master curve in Fig. 2 for a given $K$ is obtained directly from the Kramers' rate expression and not indirectly through a conversion of $p\left(F_{R}\right)$, which explains why the curves for the entire range of force could be generated from a single pulling speed $V$. However, in reality, a master curve is generated from the $p\left(F_{R}\right)$ data measured in a constant-speed SMFS experiment. ${ }^{29}$ Because of the difficulty in acquiring precise data in the tail regions of $p\left(F_{R}\right)$ (away from the mean) (see Fig. 2b-d-insets), SMFS experiments can only probe a small force range and thereby only produce a small window of data for a given $(K, V)$. Therefore, only by combining several of these windows generated from a set of $(K, V)$ is one able to generate the master curve $k(F)$ over a large range of $F$.

To conclude, unique master curves may not always be realized for arbitrary $K$ and $L$ while converting $p\left(F_{R}\right)$ from constant speed experiments to $k(F) .{ }^{29}$ Only for small $K\left(\ll 2 / \beta x^{* 2}\right)$ can one apply the soft-spring theory to $p\left(F_{R}\right)$, $k(F)$ and $\overline{F_{R}}(\dot{F})$ irrespective of $L$ and $V$. However, for large $K$, caution needs to be exercised while fitting theory, as the extracted fit-parameters will be prone to errors, especially when the window of $\dot{F}$ or $F$ is narrow.

\section{Deviation of rupture rates from soft-spring theory}

To quantify device and handle effects, we compute the deviation of the numerically determined $k_{K, L}(F)$ from soft-spring theory $k^{\text {th }}(F)$ for a given $V$ and a range of $K$ and $L$ by

$$
\varepsilon_{K, L}=\left[\sum_{i}\left|1-k_{K, L}\left(F_{i}\right) / k^{\mathrm{th}}\left(F_{i}\right)\right|\right] / \sum_{i} 1 .
$$

Here, the summation is performed within the range of forces given by the width of the distribution of rupture forces $p\left(F_{R}\right)$ about its mean, i.e. $F_{i} \in\left[\overline{F_{R}}-\sigma, \overline{F_{R}}+\sigma\right]$ where $\sigma$ refers to the standard deviation of $p\left(F_{R}\right)$. Fig. 4a summarizes the deviation (eqn (13)) at $V=10^{-10} \mathrm{~nm} / \mathrm{ps}$. Clearly, for small $K$ and large $L$ the deviations are negligible $(<5 \%)$ whereas for the largest $K$ (which is just $1.25 \times 2 / \beta x^{* 2}$ ) the deviations can be as high as $35 \%$.

To explore how the stiffness of the molecule impacts the deviations, we repeated the calculations by changing only the interaction range $x^{*}$ for a fixed $K=100 \mathrm{~kJ} \mathrm{~mol}^{-1} \mathrm{~nm}^{-1}$ and $L=100 \mathrm{~nm}$ (Fig. 4b) to find as much as $86 \%$ deviation (about 7-fold lowering of kinetics) for the shallowest energy well $\left(x^{*}=1 \mathrm{~nm}\right)$. Thus, for forced-unbinding of relatively soft molecules like RNA $\left(x^{*} \approx 10 \mathrm{~nm}^{5}\right)$ and ddFLN4 protein $\left(x^{*} \approx 1 \mathrm{~nm}^{36}\right)$, we infer lesser applicability of the soft spring model when stiff AFM tips (1-100 kJ mol $\mathrm{km}^{-1}$ ) are utilized. To test this, we use approximate data from unfolding 

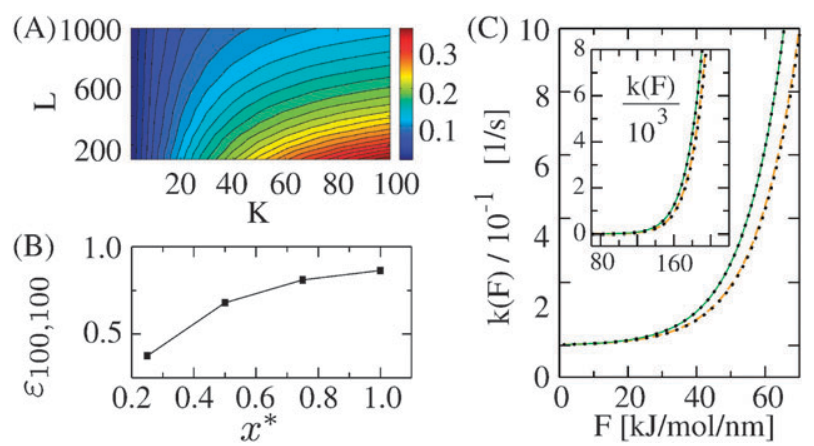

Fig. 4 (a) Magnitude of deviations $\varepsilon_{K, L}$ shown by colormap for a range of $K$ and $L$ with $V=10^{-10} \mathrm{~nm} / \mathrm{ps}$ from eqn (13). (b) Deviation $\varepsilon_{100,100}$ for a fixed $K=100 \mathrm{~kJ} \mathrm{~mol}^{-1} \mathrm{~nm}^{-2}$ and $L=100 \mathrm{~nm}$ when only the barrier distance $x^{*}[\mathrm{~nm}]$ is varied. (c),(c)-inset: Linear plots of rupture rate versus force for $(K, V)=\left(100 \mathrm{~kJ} \mathrm{~mol}^{-1} \mathrm{~nm}^{-2}, 10^{-10} \mathrm{~nm} / \mathrm{ps}\right)$ for $L[\mathrm{~nm}]=100$ (orange, dashed), 1000 (green, solid). The dotted lines are prediction from eqn (5) using $\chi \equiv \chi(F)=1+\left(K^{-1}+K_{\mathrm{wlc}}^{-1}\right)^{-1} / K_{m}$.

of ddFLN4: $:^{29,36} x^{*}=1 \mathrm{~nm} ; U^{*}=40 \mathrm{~kJ} \mathrm{~mol}{ }^{-1}$; $D=10^{-9} \mathrm{~nm}^{2} / \mathrm{ps} ; K\left[\mathrm{~kJ} \mathrm{~mol}^{-1} \mathrm{~nm}^{-2}\right]=0.05-20 ; L[\mathrm{~nm}]=$ $5-100 ; P=0.5 \mathrm{~nm} ; T=300 \mathrm{~K} ; V=2.5 \times 10^{-12} \mathrm{~nm} / \mathrm{ps}$. We find that even an AFM tip of medium stiffness, $K=20 \mathrm{~kJ} \mathrm{~mol}^{-1} \mathrm{~nm}^{-2}$, can produce discrepancies between 15 and $70 \%$ from soft spring model corresponding to handle lengths between $100 \mathrm{~nm}$ and $5 \mathrm{~nm}$.

\section{Modeling rupture rates from arbitrary device stiffness and handle lengths}

To make headway in theoretically treating $k(F)$ obtained from using arbitrary $K$ and $L$, we utilize an adapted form of eqn (5). We know for case I that $\chi$ captures the stiffness effects originating from pulling device. Extending this concept to the more general case III, we propose reparameterization of $\chi$ so as to incorporate the effective compliance of both the handle and the device:

$$
\chi \equiv \chi(F)=1+\left\{K^{-1}+K_{\mathrm{wlc}}(F)^{-1}\right\}^{-1} / K_{m}
$$

where $K_{m}$ and $K_{\text {wlc }}$ are given by eqn (12b). Such a definition of $\chi$ implicitly makes a harmonic approximation of the handle's elastic behavior at any given time, $U_{\text {han }}\left(x_{1}-x ; t\right) \approx K_{\mathrm{wlc}}(F)\left(x_{1}-x\right)^{2} / 2$ in eqn (4). This is reasonable considering that the system is instantaneously in equilibrium under quasi-static pulling speeds and when applied forces are not exceptionally high.

The rupture rate expression in eqn (5) with the reparameterized $\chi$ of eqn (14) are shown by dotted lines in Fig. 4c and its inset along with the numerically computed $k(F)$. The remarkable congruence (accurate within $3.5 \%$ for $L=100 \mathrm{~nm}$ and $K=100 \mathrm{~kJ} \mathrm{~mol}^{-1} \mathrm{~nm}^{-2}$, i.e., an order of magnitude improvement over soft-spring theory) between the two results underscores the possibility of going beyond the applicability regimes of soft-spring theory to arbitrary device stiffness and handle lengths. This framework could also be used with an FJC model or its suitable variant for tethers like PEG that are often used in conjuction with forced-rupture of ligandreceptors. In this case, however, one has to account for the force dependent compliance of the FJC linker by replacing $K_{\mathrm{wlc}}(F)^{-1}$ in eqn (14). Thus, expanding the space of observables such as $k(F)$ and $p\left(F_{R}\right)$ through designing experiments over a range of $K, L$ and $V$ will allow accurate extraction of all the key parameters: $U^{*}, x^{*}$, and $k_{0}$.

\section{Conclusion}

The theoretical framework introduced here quantifies the effects of the external elements, the pulling device and connecting handle, in single molecule force measurements. Specifically, we utilize Kramers' theory with a dynamic energy landscape description of the external elements linked to the molecule that is applicable over a broad range of pulling speeds and applied forces and not restricted to asymptotic limits. Our analyses leads us to an improved analytical model encompassing multiple control parameters (pulling speeds, handle types and lengths, device stiffnesses) that can dramatically improve prediction of activation barriers and intrinsic transition rates. The model (eqn (5)) allows for a succinct description of rupture rates in which the attributes of the handle and the device are embedded in a single parameter $\chi$ (eqn (14) and (12)). For extracting kinetic and energetic information from experimental rupture forces, we suggest conversion of $p\left(F_{R}\right)$ to $k(F)^{29}$ followed by fitting with eqn (5). Thus, force measurements from different pulling techniques can be analyzed within a common framework. Other findings of this work include derivation of physical criteria for determining when device and handle effects become important and uncovering signatures in $k(F)$ and $F_{R}(\dot{F})$ for identifying these effects.

Our approach can easily be extended to other situations. First, the effect of multiple handles and devices as well as multidomain molecules can be included. In such cases, $\chi$ will need to be modified to incorporate the compliances from the additional elements. Second, our approach uses a linear-cubic potential energy function (Fig. 1b) to describe the energetics of molecular unfolding or rupture. This choice of function imposes a condition of negligible frequency of refolding and rebinding. However, when this condition does not hold true, other energy functions such as the Morse and double-well potentials might be more appropriate. ${ }^{37}$ Finally, the worm-like chain description of the handle employed here could be replaced by other suitable constitutive models. For example, the freely-jointed chain would be a suitable model to treat polymeric handles.

\section{Abbreviations \\ SMFS single-molecule force spectroscopy \\ AFM atomic force microscope \\ WLC worm-like chain}

\section{Acknowledgements}

We thank UCSD start-up funds for supporting this work and Dr Martin Kenward for textual improvements, suggestions and graphical advice.

\section{References}

1 K. C. Neuman and A. Nagy, Nat. Methods, 2008, 5, 491.

2 J. Alegre-Cebollada, R. Perez-Jimenez, P. Kosuri and J. M. Fernandez, J. Biol. Chem., 2010, 285, 18961.

3 A. R. Bizzarri and S. Cannistraro, Chem. Soc. Rev., 2010, 39, 734. 
4 W. J. Greenleaf, K. L. Frieda, D. A. N. Foster, M. T. Woodside and S. M. Block, Science, 2008, 319, 630.

5 J. Liphardt, B. Onoa, S. B. Smith, I. Tinoco and C. Bustamante, Science, 2001, 292, 733.

6 G. I. Bell, Science, 1978, 200, 618.

7 E. Evans, D. Berk and A. Leung, Biophys. J., 1991, 59, 838.

8 A. Garg, Phys. Rev. B: Condens. Matter, 1995, 51, 15592.

9 E. Evans and K. Ritchie, Biophys. J., 1997, 72, 1541.

10 S. Izrailev, Biophys. J., 1997, 72, 1568.

11 B. Heymann and H. Grubmüller, Phys. Rev. Lett., 2000, 84, 6126.

12 H. A. Kramers, Physica, 1940, 7, 284.

13 P. Hänggi, P. Talkner and M. Borkovec, Rev. Mod. Phys., 1990, 62, 251.

14 O. K. Dudko, A. E. Filippov, J. Klafter and M. Urbakh, Proc. Natl. Acad. Sci. U. S. A., 2003, 100, 11378.

15 O. K. Dudko, G. Hummer and A. Szabo, Phys. Rev. Lett., 2006, 96, 108101.

16 R. W. Friddle, Phys. Rev. Lett., 2008, 100, 138302.

17 H. J. Lin, H. Y. Chen, Y. J. Sheng and H. K. Tsao, Phys. Rev. Lett., 2007, 98, 088304.

18 A. Maitra and G. Arya, Phys. Rev. Lett., 2010, 104, 108301.

19 R. W. Friddle, T. A. Sulchek, H. Albrecht and A. Noy, Curr. Nanosci., 2007, 3, 41.

20 E. B. Walton, S. Lee and K. J. V. Vliet, Biophys. J., 2008, 94, 2621.

21 J. D. Wen, M. Manosas, P. T. X. Li, S. B. Smith, C. Bustamante and F. Ritort, Biophys. J., 2007, 92, 2996.
22 Z. Tshiprut, J. Klafter and M. Urbakh, Biophys. J., 2008, 95, L42.

23 C. Friedsam, A. K. Wehle, F. Kühner and H. E. Gaub, J. Phys.: Condens. Matter, 2003, 15, S1709.

24 T. V. Ratto, K. C. Langry, R. E. Rudd, R. L. Balhorn, M. J. Allen and M. W. McElfresh, Biophys. J., 2004, 86, 2430.

25 F. Kühner and H. E. Gaub, Polymer, 2006, 47, 2555.

26 C. Hyeon and D. Thirumalai, Biophys. J., 2006, 90, 3410.

27 E. Evans and K. Ritchie, Biophys. J., 1999, 76, 2439.

28 C. Ray, J. R. Brown and B. B. Akhremitchev, J. Phys. Chem. B, 2007, 111, 1963.

29 O. K. Dudko, G. Hummer and A. Szabo, Proc. Natl. Acad. Sci. U. S. A., 2008, 105, 15755.

30 C. Bustamante, J. Marko, E. Siggia and S. Smith, Science, 1994, 265, 1599.

31 J. F. Marko and E. D. Siggia, Macromolecules, 1995, 28, 8759.

32 L. B. Freund, Proc. Natl. Acad. Sci. U. S. A., 2009, 106, 8818.

33 M. Abramowitz and I. Stegun, Handbook of Mathematical Functions, Dover, New York, 1972.

34 Z. Tshiprut, A. E. Filippov and M. Urbakh, J. Phys.: Condens. Matter, 2008, 20, 354002.

35 C. Jarzynski, Eur. Phys. J. B, 2008, 64, 331.

36 M. Schlierf and M. Rief, Biophys. J., 2006, 90, L33.

37 R. Berkovich, S. Garcia-Manyes, M. Urbakh, J. Klafter and J. M. Fernandez, Biophys. J., 2010, 98, 2692. 